\title{
4-year optical-infrared photometric and polarimetric behaviors of a gamma- ray blazar 3C 454.3
}

\author{
M. Sasada ${ }^{1, a}$, M. Uemura ${ }^{2}$, Y. Fukazawa ${ }^{3}$, R. Itoh ${ }^{3}$, K. Kawabata ${ }^{2}$, and M. Yoshida ${ }^{2}$ \\ ${ }^{1}$ Kyoto University, Kitashirakawa-Oiwake-cho, Sakyo-ku, Kyoto, Japan 606-8502 \\ ${ }^{2}$ Astrophysical Science Center, Hiroshima University, Kagamiyama 1-3-1, Higashi-Hiroshima, Hiroshima, Japan \\ ${ }^{3}$ Department of Physical Science, Hiroshima University, Kagamiyama 1-3-1, Higashi-Hiroshima, Hiroshima, Japan
}

\begin{abstract}
.
We have performed a multi-color photo-polarimetric monitoring of 45 optical-bright blazars since 2007 . 3C 454.3, which is one of the brightest blazars in the gamma-ray sky, is one of targets of its project. We have made photo-polarimetric monitorings since July 2007. The object showed violent variation in its flux and polarization. In particular, four large-amplitude outbursts in 2007, 2008, 2009 and 2010 are remarkable. In each outburst, we observed a significant rise in the polarization degree. We calculated structure functions of the total flux and polarization from the 4-year monitoring data. In this time, we estimate the systematic and statistical errors of its structure functions. We adopt these errors when we estimate the break time-scales. The break timescale of the total flux in 3C 454.3 is longer than that of the polarization. We also calculate the structure functions from total flux and polarization of the 15 blazars which are high-frequency monitored, and estimate the break time-scales. Most of estimated break time-scales of the total fluxes are longer than that of the polarization. From these results, it is indicated that the break time-scale of the total flux is reflected to the larger-amplitude and longer variation, like the outburst. However, the break time-scale of the polarization is related to the time-scale of the day-scale flares.
\end{abstract}

\section{Introduction}

Relativistic jets are often associated with active galactic nuclei (AGNs). A blazar is a subclass of AGN with relativistic jets, which is widely believed to be viewed at small angle to the line of sight. A blazar often shows violent variations in a flux and polarization. These variations show variety time-scales from minutes [e.g. 3, 11] to years [e.g. 15]. Blazars sometimes become 10 times or more brighter than its quiescent states. This brightening phenomenon is often called as an outburst. Its variation behaviors of the flux and polarization are complex, and thus, continuous and long-term photometric and polarimetric monitorings are needed.

$3 C 454.3$, redshift is $z=0.859$, is one of the most famous blazars by occurring with large-amplitude outbursts. In the outburst, the object became bright in all frequency from radio to gamma-ray bands. The object has showed dramatic outbursts in 2005 (e.g. [5]), 2007 (e.g. [18]), 2008 (e.g. [1]), 2009 (e.g. [10]) and 2010 (e.g. [2, 19]), respectively. In these outbursts, the polarization also showed the violent variations in the radio and optical bands [9, 12, 13].

In this paper, we report the results of the optical and near-Infrared (NIR) photopolarimetric monitoring of 3C 454.3 for 4 years including four outbursts using the Kanata telescope. We also report the variations of total

\footnotetext{
a. e-mail: sasada@kusastro.kyoto-u.ac.jp
}

flux and polarization in 14 other high-frequency monitored blazars in our blazar-monitoring project.

\section{Observation}

We performed multi-band photopolarimetric monitorings to 45 blazars in the optical and NIR bands using TRISPEC [20] attached to the 1.5-m Kanata telescope at Higashi-Hiroshima Observatory. TRISPEC can perform photopolarimetric observations in the optical and two NIR bands, simultaneously. Unfortunately, one of the two NIR arrays sometimes was not available due to readout errors. We, thus, mainly obtained the photopolarimetric data in the $V$ and $J$ bands. A unit of the observing sequence consisted of successive exposures at four position angles of a half-wave plate. A polarization was derived from each set of four exposures.

We changed the integration time day by day depending on the sky condition and brightness of the objects. All images were bias-subtracted and flat-fielded before performing aperture photometry. We performed differential photometry with comparison stars taken in the same frames of the objects. The comparison stars refer to Ikejiri et al. [7]. We adopt Galactic extinctions of the objects in the $V$ and $J$ bands from Schlegel et al. [14]. We converted into fluxes using the fluxes at 0 magnitudes of $V$ and $J$ bands [4, 6]. 
We confirmed that the instrumental polarization of TRISPEC was smaller than $0.1 \%$ in the $V$ and $J$ bands using the unpolarized standard stars. Thus, we did not apply the correction for the instrumental polarization. We applied the instrumental depolarization factors as 0.827 and 0.928 in the $V$ and $J$ bands, respectively. The zero point of the PA is defined by the standard system (measured from north to east) by observing the polarized standard stars, HD 19820 and HD 25443 [21].

\section{Result}

\section{$3.13 C 454.3$}

We have perfored the $V$ - and $J$-band photopolarimetric monitoring to the gamma-ray detected blazar 3C 454.3 for 4 years since July 2007. The result of this monitoring is referred to Sasada et al. [12 13]. Figure 1 shows the variations of $V$-band total and polarized fluxes, degree and angle of polarization, and spectral index, $\alpha_{V J}$; $F_{v} \approx v^{-\alpha}$, between the $V$ and $J$ bands in the object. The object showed several outbursts in which flux is increased with month time-scales in every years, and a lot of daytime-scale flares. The polarized flux also showed violent variability. The increases of degree of polarization is associated with the large-amplitude flux increases during its outbursts. The $\alpha_{V J}$ became flat when the object became faint. This is caused by the increase of the fraction of the thermal radiation from the accretion disk which peak is in the UV band against the synchrotron radiation.

\subsection{Structure Function}

A structure function (SF) is equivalent to the power density spectrum (PDS) of the flux calculated in the time domain instead of frequency space, which makes it less subject to sampling problems in the irregular time series (see e.g. [16]). The definition of SF in the flux variation $(F(t))$ is calculated from the mean squared of fluxes of different times between $t$ and $t+\Delta \tau$ of $N$ pairs. The first-order $S F$ of the flux, $\mathrm{SF}_{\mathrm{F}}$, is represented as ;

$$
\mathrm{SF}_{\mathrm{F}}^{(1)}(\Delta \tau)=\frac{1}{N} \sum_{i=1}^{N}\left[F\left(t_{i}\right)-F\left(t_{i}+\Delta \tau\right)\right]^{2} .
$$

In the case of linear polarization, there are two parameters, Stokes parameters $Q$ and $U$. We define the moving distance of the polarization vector, $l$, and estimate the SF about the polarization using the parameter $l$. The $l$ is the distance between the polarizations of different times in $t$ and $\Delta \tau$, and is represented as ;

$$
\begin{aligned}
l_{i}^{2}(\Delta \tau)=\quad\{ & \left.Q\left(t_{i}\right)-Q\left(t_{i}+\Delta \tau\right)\right\}^{2} \\
& +\left\{U\left(t_{i}\right)-U\left(t_{i}+\Delta \tau\right)\right\}^{2},
\end{aligned}
$$

where $Q(t)$ and $U(t)$ are the Stokes parameters in the flux unit. Using the distance of polarization, we can estimate the $\mathrm{SF}$ of the polarization, $\mathrm{SF}_{\mathrm{P}}$, as follow;

$$
\mathrm{SF}_{\mathrm{P}}^{(1)}(\Delta \tau)=\frac{1}{N} \sum_{i=1}^{N} l_{i}^{2}(\Delta \tau) .
$$

There are breaks in the $\mathrm{SF}_{\mathrm{F} / \mathrm{P}}$. These break times $\tau_{\mathrm{br}, \mathrm{F} / \mathrm{P}}$ should indicate the typical variation time-scales of the flux and polarization. We can know the typical variation timescales by estimating the $\tau_{\mathrm{br}}$. We estimate the $\tau_{\mathrm{br}}$ by fitting a knee model. The knee model is represented as;

$$
\operatorname{SF}_{\mathrm{F} / \mathrm{P}}(\Delta \tau)=\frac{a}{\left[1+\left(\frac{\Delta \tau}{\tau_{\mathrm{br}}}\right)^{-b}\right]^{c / b}}
$$

where $a$ is the normalization of the SF, $b$ is an index of sharpness at the $\tau_{\mathrm{br}}$ of the SF, and $c$ is an slope index before the $\tau_{\mathrm{br}}$. If the $b$ is large, the bentness of SF at the $\tau_{\mathrm{br}}$ is sharp. And if the $c$ is large, the slope of SF before the $\tau_{\text {br }}$ is steep. When the knee-model function is fitted to the data, the error of the SF is needed.

In order to estimate the total error $\sigma$, we should estimate the systematic and statistical errors, $\sigma_{\text {sys }}$ and $\sigma_{\text {sta. }}$. In the case of optical monitoring, the observing time intervals should be irregular, because of the day time, sky conditions, or machine time limit, etc. Thus, the calculated SF have systematic uncertainty caused by the irregular observing intervals. We estimate this uncertainty as the $\sigma_{\text {sys }}$ by using the Monte Carlo method. We conform to the procedure written in Iyomoto et al. [8] in order to estimate the $\sigma_{\text {sys }}$. We assume a power spectrum density (PSD) as a broken powerlaw. At this time, we should assume the break frequency and powerlaw index. We generate 1000 simulated light curves using the assumed PSD, which generating procedure is referred to Timmer \& König [17]. We select the data points of simulated light curves same as the observing intervals. At this time, we normalize the simulated light curves using the standard deviation of the observed light curve. After the normalization, we calculate the SFs of the extracted and normalized light curves. After 1000 times trials, we calculate the average and standard deviation of the simulated SFs. We estimate a chi-square from the observed, and averaged simulated SFs, and standard deviation of simulated 1000 SFs. We estimate the chisquares of assumed PSD with various parameter pairs. The assumed PSD with the minimum chi-square is the most suitable PSD for the observed light curve. The standard deviation calculated from this PSD is regarded as the $\sigma_{\text {sys }}$ of the observing intervals.

We also estimate the $\sigma_{\text {sta }}$ of the SF. Each point of light curve has an uncertainty as a statistical error estimated from a deviation of the data. We estimate the $\sigma_{\text {sta }}$ of SF caused by the uncertainties of individual data by using a bootstrap method. The uncertainty of each point of light curve is equal to the half width hull maximum (FWHM) of the probability distribution which can be described as the Gauss function. We generate a pseudo light curve calculated from random numbers following the Gaussian distribution with the average and FWHM which are the same value as the observed data point and its error. We calculate the SF from the pseudo light curve. We try 1000 times simulations, and estimate its average and standard deviation. The standard deviation is regarded as the $\sigma_{\text {sta }}$ of the observed SF. The sum of the $\sigma_{\text {sys }}$ and $\sigma_{\text {sta }}$ is regarded as the total error, $\sigma$, of the SF. 


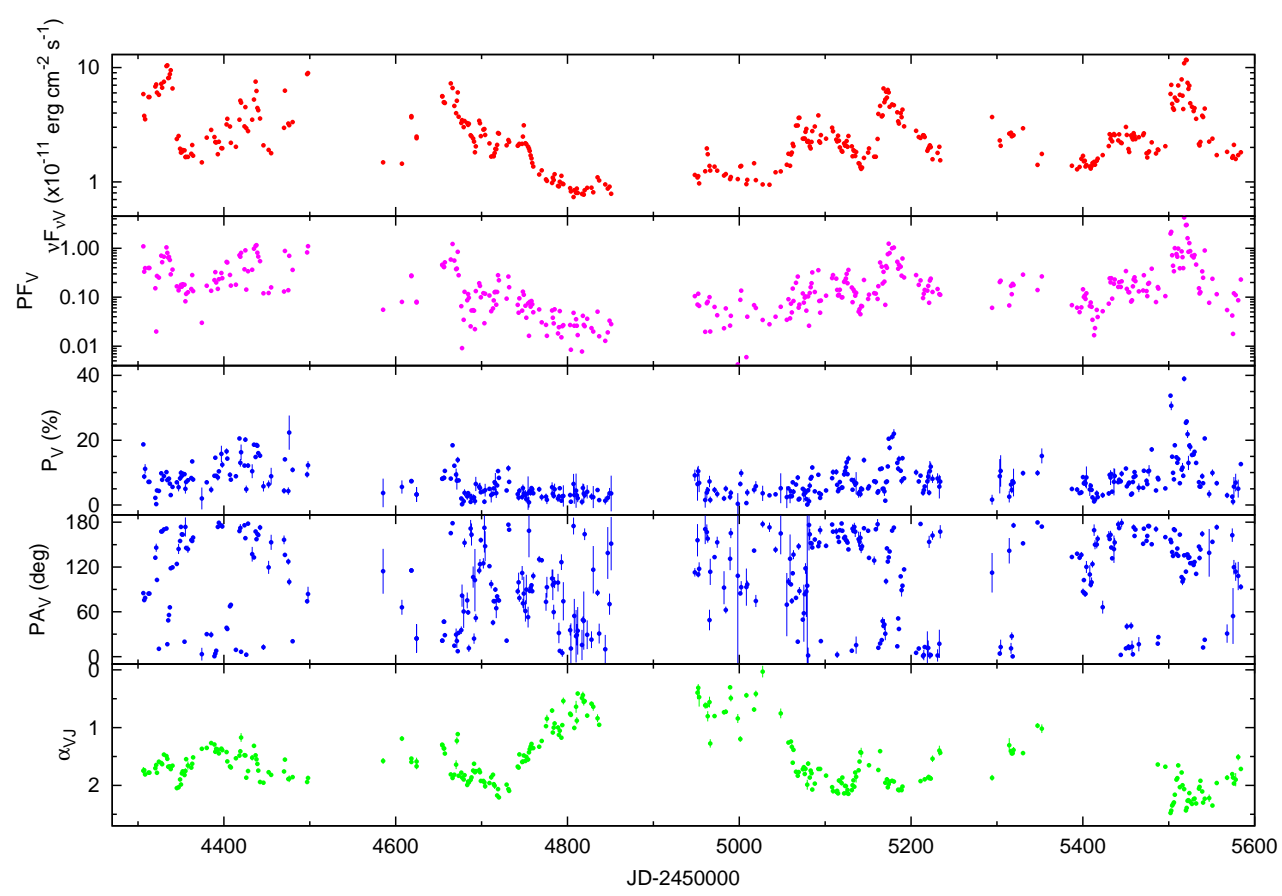

Figure 1. Variation parameters of $3 \mathrm{C} 454.3$. From top to bottom panels, we show the $V$-band light curve, polarized flux variation, variations of degree and angle of polarization, and spectral index between the $V$ and $J$ bands.

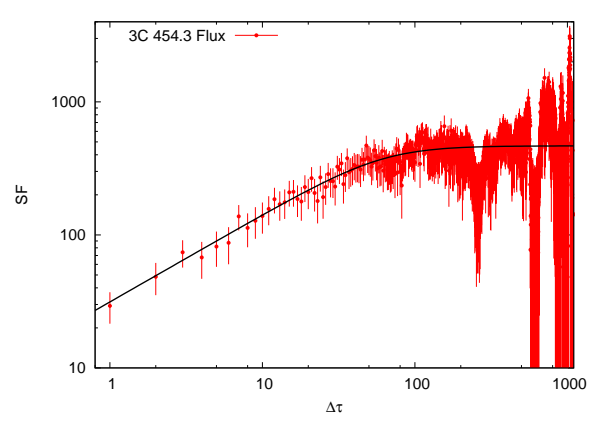

Figure 2. SF of the total flux. We also show the best fitted function as a solid line.

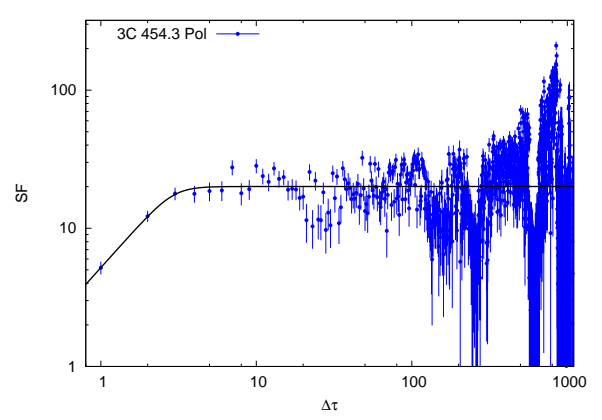

Figure 3. SF of the polarization. We show the best fitted function as a solid line.
Figure 2 and 3 show the SFs of the total flux and polarization of 3C 454.3. We also show the solid lines with the best fitted parameters of the knee model estimated by Markov chain Monte Carlo (MCMC) methods. In the MCMC methods, we estimate $\tau_{\mathrm{br}}, a$, and $c$ as free parameters. Then, we fix the $b$ to 2 or 4 . Estimated $\tau_{\text {br }} \mathrm{s}$ in the SFs of the total flux and polarization are $61_{-8}^{+9}$ days and $2.9 \pm 0.2$ days, respectively. The $\tau_{\mathrm{br}, \mathrm{F}}$ and $\tau_{\mathrm{br}, \mathrm{P}}$ are apparently different.

\section{Apply to 15 Blazars}

We have performed 45 blazar monitorings since 2007 using Kanata/TRISPEC (see Ikejiri et al. [7] for details). The numbers of the monitoring dates of our targets are different. These differences are depending on positions of the targets, sky conditions, its brightnesses and so on. All blazars show the flux and polarization variations and some of our targets showed large-amplitude variations like outbursts. We calculate structure functions of the total flux and polarization to other 14 high-frequency monitored blazars in our project.

We calculate the $\mathrm{SF}_{\mathrm{F}}$ and $\mathrm{SF}_{\mathrm{P}}$ of 14 blazars which numbers of data are larger than 70 points in order to estimate more accurate SFs. We also estimate the total errors of the calculated SFs, and estimate the $\tau_{\mathrm{br}, \mathrm{F}}$, and $\tau_{\mathrm{br}, \mathrm{P}}$ of SF in each object. We estimate both $\tau_{\mathrm{br}, \mathrm{F}}$ and $\tau_{\mathrm{br}, \mathrm{P}}$ of 10 blazars out of 14 blazars. 4 blazars do not show the break in the SFs.

Figure 4 shows the relation between the $\tau_{\mathrm{br}, \mathrm{F}}$ and $\tau_{\mathrm{br}, \mathrm{P}}$ of 11 blazars including 3C 454.3. The solid line shows the unity relation of these $\tau_{\mathrm{br}}$. The $\tau_{\mathrm{br}, \mathrm{P}} \mathrm{s}$ of most of blazars 


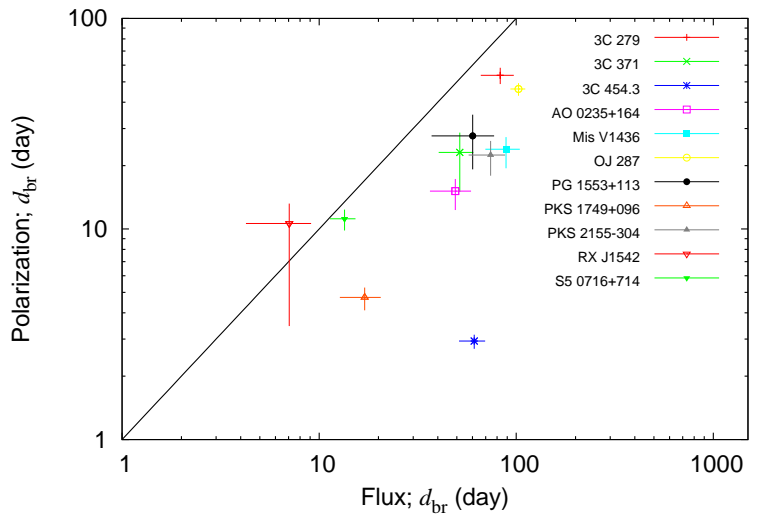

Figure 4. Relation between the $\tau_{\mathrm{br}, \mathrm{F}}$ and $\tau_{\mathrm{br}, \mathrm{P}}$ of 11 blazars. A solid line shows the unity relation of the $\tau_{\mathrm{br}, \mathrm{F}}$ and $\tau_{\mathrm{br}, \mathrm{P}}$.

are shorter than the $\tau_{\mathrm{br}, \mathrm{F}}$. There is no object which $\tau_{\mathrm{br}, \mathrm{P}}$ is longer than $\tau_{\mathrm{br}, \mathrm{F}}$ over the error ranges. This fact that the flux variation is longer than or at least equal to the polarization variation.

\section{Discussion}

The $\tau_{\mathrm{br}, \mathrm{F}} \mathrm{s}$ of the high-frequency monitored blazars, which indicate the flux variation time-scales, are always longer than or equal to the $\tau_{\mathrm{br}, \mathrm{P}} \mathrm{s}$. In order to explain this result, we suggest the blazar has several emission regions.

If there are two emission regions in the jet of a blazar, the observed flux is simply summing these emissions. Then, the variation time-scale should be longer. However, in the case of polarization, these two polarizations should be summed vectorially. At this time, the two polarizations should diminish each other at the overlapping range, unless the directions of the polarizations are the same. Then, the variation time-scale of the polarization becomes equal or shorter. Thus, the variation time-scale of the polarization seems to be reflected to the physical time-scale of the emitting region.

The $\tau_{\text {br }}$ of SF is strongly influenced by the largeramplitude variations. Thus, the large-amplitude outburst is more affected to the SF of the total flux than the individual flares. The $\tau_{\mathrm{br}, \mathrm{F}}$ of $3 \mathrm{C} 454.3$ may show the timescale of its outbursts. On the other hand, the $\tau_{\text {br,P }}$ of the object seems to be reflected to the time-scale of its individual flares, because of the diminishing each other. Other blazars also showed the similar trend between its $\tau_{\mathrm{br}, \mathrm{F}}$ and $\tau_{\text {br,P. }}$ As a result, the $\tau_{\text {br,F }}$ is reflected to the large-amplitude and longer time-scale variations, like an outburst. On the other hand, the $\tau_{\mathrm{br}, \mathrm{P}}$ is reflected to the day-scale flares.

\section{Conclusion}

3C 454.3 showed the flux and polariation variations in our monitoring. The object showed large-amplitude and longer-time-scale outbursts, and day-scale flares. We calculate structure functions about the total flux and polarization, and estimate the break time-scales as the variation time-scales. Then, the break time-scale of the total flux is longer than that of the polarization. This trend was shown in other high-frequency monitored blazars.

\section{References}

[1] Abdo, A. A., et al. 2009, ApJ, 699, 817

[2] Abdo, A. A., et al. 2011, ApJ, 733, L26

[3] Aharonian, F., et al. 2007, ApJ, 664, L71

[4] Bessell, M. S., Castelli, F., \& Plez, B. 1998, A\&A, 333,231

[5] Fuhrmann, L., et al. 2006, A\&A, 445, L1

[6] Fukugita, M., Shimasaku, K., \& Ichikawa, T. 1995, PASP, 107, 945

[7] Ikejiri, Y., et al. 2011, PASJ, 63, 639

[8] Iyomoto, N., \& Makishima, K. 2001, MNRAS, 321, 767

[9] Jorstad, S. G., Marscher, A. P., Larionov, V. M., Agudo, I., Smith, P. S. et al. 2010, ApJ, 715, 362

[10] Pacciani, L., et al. 2010, ApJ, 716, L170

[11] Sasada, M., et al. 2008, PASJ, 60, L37

[12] Sasada, M., et al. 2010, PASJ, 62, 645

[13] Sasada, M., et al. 2012, PASJ, 64, 58

[14] Schlegel, D. J., Finkbeiner, D. P., \& Davis, M. 1998, ApJ, 500, 525

[15] Sillanpää, A., et al. 1996, A\&A, 305, L17

[16] Simonetti, J. H., et al. 1985, ApJ, 296, 46

[17] Timmer, J., and König M., 1995, A\&A, 300, 707

[18] Vercellone, S., et al. 2008, ApJ, 676, L13

[19] Vercellone, S., et al. 2011, ApJ, 736, L38

[20] Watanabe, M., et al. 2005, PASP, 117, 870

[21] Wolff, M. J., Nordsieck, K. H., \& Nook, M. A. 1996, AJ, 111, 856 